\title{
Antipsychotika bei Demenz
}

\author{
Jürgen Fritze, Josef Aldenhoff, Frank Bergmann, Wolfgang Maier, Hans-Jürgen Möller
}

für die Deutsche Gesellschaft für Psychiatrie, Psychotherapie und Nervenheilkunde (DGPPN) und die Arbeitsgemeinschaft für Neuropsychopharmakologie und Pharmakopsychiatrie (AGNP)

psychoneuro 2005; 31 (11): 581-583

S eit über drei Jahren wird eine erhöhte Mortalität älterer, demenzkranker Menschen unter der Therapie von Verhaltensstörungen mit atypischen Antipsychotika diskutiert. Im April 2005 hat die Food and Drug Administration (2) die pharmazeutischen Unternehmer aufgefordert, in die Fachinformationen aller atypischen Neuroleptika, also Aripiprazol (Abilify ${ }^{\circledR}$ ), Olanzapin (Zyprexa ${ }^{\circledR}$ ), Quetiapin (Seroquel ${ }^{\circledR}$ ), Risperidon (Risperdal $^{\circledR}$ ), Clozapin (Leponex ${ }^{\circledR}$, Elcrit ${ }^{\circledR}$, Generika) und Ziprasidon (Zeldox ${ }^{\circledR}$ ) einen Warnhinweis bezüglich einer erhöhten Mortalität dieser Patienten im Vergleich zu Plazebo bei fehlender („off-label-use“) Zulassung (in Deutschland ist Risperidon in dieser Indikation zugelassen) aufzunehmen. Die FDA begründete ihre Forderung damit, dass in einer zusammenfassenden Analyse von 17 plazebokontrollierten Untersuchungen von vier der o.g. Wirkstoffe (Aripiprazol, Olanzapin, Quetiapin, Risperidon) an insgesamt $5106 \mathrm{~Pa}-$ tienten die Sterblichkeit 1,6- bis 1,7fach über der unter Plazebo (4,5\% versus 2,6\%) lag. Der Unterschied des absoluten Risikos (1,9\%) entspricht einer $\mathrm{NNH}$ (number needed to harm) von 52; danach wäre also mit einem dem atypischen Neuroleptikum anzulastenden Todesfall bei 52 behandelten Patienten zu

Aus einem Warnhinweis der FDA ist zu schlussfolgern, dass atypische Neuroleptika als Klasse ein erhöhtes Mortalitätsrisiko bei älteren Patienten bergen, solange sich aus einer unabhängigen Re-Analyse der Daten nichts anderes ergibt. Eine Re-Analyse ist derzeit unmöglich. Da es sich um eine häufige Nebenwirkung handelt, ist sie beim Einholen des informierten Einverständnisses zu berücksichtigen. Da die FDA auch bei typischen Neuroleptika ein ähnliches Risiko für möglich hält, gilt dies auch hier.

rechnen. Dabei ist zu beachten, dass die zugrunde liegenden Studien Beobachtungszeiten von maximal drei Monaten hatten.

In 15 dieser Studien war die Sterblichkeit nominal erhöht. Die Todesursachen hingen überwiegend mit Herzerkrankungen (u.a. Herzversagen, plötzlicher Tod) oder Infektionen (überwiegend Pneumonien) zusammen. Die FDA bewertet dies als Klasseneffekt, erwägt aber, den Warnhinweis auf alle Neuroleptika zu erweitern. Nachdem bereits im Jahr 2004 u.a. Arzneimittelbehörden und Hersteller auf ein erhöhtes Risiko zerebrovaskulärer Ereignisse speziell unter Risperidon und Olanzapin und später auch Aripiprazol im Vergleich zu Plazebo hingewiesen hatten, haben Jüptner \& Gastpar (6) im Auftrag der DGPPN und AGNP zu dieser Frage Stellung genommen und dabei insbesondere darauf hingewiesen, dass die Rate zerebrovaskulärer Ereignisse nicht nur unter diesen beiden Wirkstoffen erhöht beobachtet wurde, sondern auch unter typischen Neuroleptika wie Haloperidol und unter Benzodiazepinen bei insgesamt allerdings schwacher Datenlage. Die Diskussion hat zur Verunsicherung geführt, wie mit dem klinisch bedeutsamen Problem der Verhaltensstörungen bei Demenz umzugehen sei. Jüptner \& Gastpar (6) haben konsistente Hinweise gegeben: Zunächst sei die günstige Wirkung von Cholinesterasehemmern auch auf Verhaltensstörungen zu nutzen, nur im zweiten Schritt seien Neuroleptika einzusetzen und dies nur gezielt, streng indiziert und nur vorübergehend. In der Zwischenzeit ist zumindest eine weitere, allerdings retrospektive Kohortenstudie ( $\mathrm{n}=17845$ unter atypischen Neuroleptika, $\mathrm{n}=14865$ unter typischen) publiziert worden (3), wonach das Risiko ischämischer Hirninfarkte sich zwischen typischen und atypischen Neuroleptika nicht unterscheidet. Da die Studie auf Datenbanken basierte, konnten allerdings nur Ereignisse erfasst werden, die zu Hospitalisierung führten. Herrmann \& Lanctot (5) identifizierten eine Reihe methodi- 


\section{Tab. 1 Todesfälle bei älteren Patienten mit Demenz in plazebo- kontrollierten Studien}

\begin{tabular}{|c|c|c|c|}
\hline Antipsychotikum & $\begin{array}{l}\text { Anzahl } \\
\text { Studien }\end{array}$ & $\begin{array}{c}\text { Todesfälle: alle Ursachen/Anzahl } \\
\text { behandelter Patienten }\end{array}$ & Prozent (\%) \\
\hline $\begin{array}{l}\text { Clozapin } \\
\text { Plazebo }\end{array}$ & $*$ & $*$ & * \\
\hline Risperidon & 6 & $40 / 1009$ & 4,0 \\
\hline Plazebo & & $22 / 712$ & 3,1 \\
\hline Quetiapin & 2 & $20 / 365$ & 5,51 \\
\hline Plazebo & & $7 / 217$ & 3,2 \\
\hline Olanzapin & 5 & $42 / 1184$ & 3,5 \\
\hline Plazebo & & $7 / 478$ & 1,5 \\
\hline
\end{tabular}

scher Schwächen der Untersuchungen von Risperidon bzw. Olanzapin, die den Warnhinweisen der Arzneimittelbehörden zugrunde lagen.

An die DGPPN wurden nun erneut Fragen herangetragen, wie angesichts des anscheinend bei allen Neuroleptika erhöhten Risikos insbesondere aggressive Verhaltensstörungen Demenzkranker zu behandeln seien, wie über etwaige Risiken auch unter konventionellen Neuroleptika, hier insbesondere Melperon (z.B. Eunerpan ${ }^{\circledR}$ ) und Pipamperon (Dipiperon ${ }^{\circledR}$ ) aufzuklären sei, ob ggf. ein schriftliches Einverständnis des Betreuers einzuholen sei, welche Anforderungen an die Einwilligung im Notfall zu stellen seien, und welche haftungsrechtlichen Folgen drohen.

Die dem jüngsten Warnhinweis zugrunde liegenden Studien hat die FDA nicht spezifiziert. Sink et al. (7) konnten in ihrer systematischen Übersicht nur sechs publizierte Studien zu atypischen Neuroleptika identifizieren, so dass die FDA vermutlich auch auf unpublizierte, ihr im Rahmen von Zulassungsverfahren vorgelegte Studien zurückgegriffen hat. Dies bestätigten jüngst (13.07.2005) Singh \& Wooltorton (8). Deshalb muss derzeit eine unabhängige Validierung der Daten weitgehend scheitern. Immerhin präsentieren Singh \& Wooltorton (8) die Daten aus 13 der Studien, die Health Canada (4) am 22.06.2005 aus dem „Canadian Adverse Drug Reaction Monitoring Program (CADRMP)“ im Internet (jetzt an u.g. Adresse) veröffentlicht hatte (Tab. 1). Eine tiefergehende Analyse ist unmöglich.
Auch ist zu bedenken, dass die Nebenwirkungsereignisse quantitativ überschätzt werden können, da Studienabbrüche in plazebokontrollierten Studien überzufällig häufig unter der Plazebobedingung auftreten und die Beobachtungszeiten somit verkürzt sind, so dass eine Unterschätzung der Ereignisraten in der Plazebogruppe resultieren kann.

Ein erhöhtes Risiko muss aber als gegeben akzeptiert werden. Der Warnhinweis der FDA und der kanadischen Behörde ist auch in Deutschland ernst zu nehmen, auch wenn die deutsche Behörde bisher abgesehen von den Sicherheitshinweisen aus dem Jahr 2004 zu erhöhtem Risiko für das Auftreten von Schlaganfällen, einschließlich solcher mit tödlichem Verlauf, bei älteren Patienten mit Demenz-bedingten Verhaltensstörungen unter Risperidon und Olanzapin - nichts Vergleichbares verlautbart hat.

Dieser Sachstand ändert nichts an den Aussagen von Jüptner \& Gastpar (6). Deren Schlussfolgerung, dass auch von typischen Neuroleptika erhöhte Risiken ausgehen können, wird grundsätzlich bestätigt, wenn auch endgültige Aussagen der FDA ausstehen. Gemäß der systematischen Übersicht von Sink et al. (7) existieren zu typischen Neuroleptika nur 14 publizierte plazebokontrollierte Studien (insgesamt $n=1237$ ). Über die Sterblichkeitsraten geben sie keine detaillierten Informationen. In diesen wurde überwiegend Haloperidol untersucht, ansonsten Thioridazin, Perphenazin, Chlorpromazin, Thiothixen (in BRD nicht verfügunter Plazebobedingung im Mittel bar), Trifluoperazin (in BRD nicht mehr verfügbar), Acetophenazin (in BRD nicht verfügbar). Die Agency for Healthcare Research and Quality (AHRQ 2004) konnte in ihrem HTAReport für Melperon keine plazebokontrollierten Studien identifizieren, Pipamperon wird im HTA-Report nicht einmal erwähnt. Spezifische Aussagen über die Risiken einzelner typischer Neuroleptika werden also kaum möglich sein; die Aussagen der FDA bleiben abzuwarten.

Die Wirksamkeit gegen Verhaltensstörungen bei Demenz ist für atypische Neuroleptika deutlich besser als für typische belegt (7). Die Wirksamkeit ist allerdings begrenzt. Die Responderraten liegen nur 16\% bis $26 \%$ über Plazebo. Entsprechend empfehlen z.B. Sink et al. (7), zunächst nicht-pharmakologische Therapie anzuwenden; dazu gehört das Training der Bezugspersonen im Umgang mit den Demenzkranken. Von Antidepressiva ist - abgesehen von der antidepressiven Wirkung keine Besserung der Verhaltensstörungen zu erwarten; nur für Citalopram liegt eine positive Studie vor. In drei plazebokontrollierten Studien war Valproinsäure unwirksam (7). Die Ergebnisse der beiden Studien zu Carbamazepin sind widersprüchlich. Benzodiazepine sollten generell in dieser Patientengruppe wegen Sedierung, Sturzgefahr, Verschlechterung der Kognition und Abhängigkeitspotential vermieden werden.

Sofern nicht bereits bestehend, sollten im nächsten Schritt Cholinesterasehemmer eingesetzt werden, von denen - wenn auch in begrenztem Maße (9) - eine Besserung auch der nicht-kognitiven Störungen erwartet werden kann (7). Wenn Antipsychotika unerlässlich sind, insbesondere bei psychotischen Symptomen, so sind - wie von Jüptner \& Gastpar (6) bereits gut begründet atypische Neuroleptika insbesondere wegen ihrer besseren extrapyramidalmotorischen Verträglichkeit gegenüber typischen Neuroleptika zu bevorzugen, hier angesichts des Zulassungsstatus und der besten Datenlage insbesondere Risperidon. Der Warnhinweis der FDA bezüglich erhöhter Mortalität hat grundsätz- 
lich keine spezifischen Konsequenzen für das Verordnungsverhalten. Jeder Off-Label-Use bedarf in jedem Einzelfall einer besonderen Abwägung der Risiken der unbehandelten Störung gegen die Risiken der antipsychotischen Pharmakotherapie einschließlich detaillierter Dokumentation. Im Einzelfall kann sogar eine Verpflichtung zum Off-LabelUse bestehen. Je geringer die verfügbare Evidenz für einen Nutzen des Off-Label-Use ist, desto weniger ist dieser gerechtfertigt. Dies gilt nicht nur haftungsrechtlich, sondern - vor dem Hintergrund des Urteils des Bundessozialgerichtes - auch sozialrechtlich. Die Ätiologie (Alzheimer, vaskulär etc.) der Demenz spielt dabei keine spezifische Rolle, da die Indikation von Risperidon syndromal formuliert ist. Die verfügbare Evidenz ist besser für die atypischen Neuroleptika als für die typischen (7). Außerdem ist die Verträglichkeit besser, im Alter von besonderer Relevanz. Ein erhöhtes Mortalitätsrisiko auch unter typischen Neuroleptika ist möglich. Es gibt keine Hinweise, dass dennoch typische Neuroleptika diesbezüglich sicherer als atypische wären. All das spricht für ein Präferieren der atypischen.

Über die erhöhte Mortalität unter atypischen Neuroleptika als Klasseneffekt und die Abwägung der Alternativen (z.B. die Risiken und Nebenwirkungen der typischen Neuroleptika) ist als - in Begriffen der Arzneimittelsicherheit - häufige (1-10\%) Nebenwirkung aufzuklären, soweit dem Demenzkranken zumutbar, ansonsten ggf. ergänzend oder ersatzweise der Betreuer. Um Beweislastumkehr zu vermeiden, ist entsprechend $\mathrm{zu}$ dokumentieren. Ob das Einverständnis schriftlich oder mündlich eingeholt wird, liegt im subjektiven Ermessen und mag von Fall zu Fall variieren. Wie immer sind bei sachgerechter Aufklärung und Dokumentation keine spezifischen Haftungsprobleme $\mathrm{zu}$ erwarten.

\section{Atypical neuroleptics in dementia} A warning of the FDA has to be accepted as meaning that atypical neuroleptics as a class increase the death rate in elderly patients as long as there is no independent re-analysis yielding different conclusions. At present, a re-analysis is impossible. As this is a frequent adverse reaction, it has to be considered when seeking informed consent. As the FDA suggests a similar risk for typical neuroleptics, this is also true for these drugs.

\section{Key Words}

atypical neuroleptics - dementia risks

\section{Literatur:}

1. Agency for Healthcare Research and Quality: Pharmacological Treatment of Dementia. Evidence Report/Technology Assessment, Number 97. AHRQ Publication No. 04-E018-2, April 2004

2. FDA. FDA Public Health Advisory Deaths with Antipsychotics in Elderly Patients with Behavioral Disturbances. http://www.fda.gov/cder/drug/advisory/ antipsychotics.htm

3. Gill SD, Rochon PA, Herrmann N, Lee PE, Sykora K, Gunraj N, Normand S-LT, Gurwitz $\mathrm{JH}$, Marras C, Wodchis WP, Mamdani M. Atypical antipsychotic drugs and risk of ischaemic stroke: population based retrospective cohort study. BMJ 2005; 330: 445450

4. Health Canada. Increased Mortality Associated with the Use of Atypical Antipsychotic Drugs in Elderly Patients with Dementia. http://www.hc-sc.gc.ca/dhp-mps/ medeff/advisories-avis/prof/atyp-anti psycho hpc-cps e.html

5. Herrmann N, Lanctot KL. Do atypical antipsychotics cause stroke? CNS Drugs 2005; 19: 91-103

6. Jüptner M, Gastpar M. Todesfälle unter Risperidon und Olanzapin - was nun? - Behandlung psychotischer Symptome bei Demenzpatienten. psychoneuro 2004; 30 : 314-316

7. Sink KM, Holden KF, Yaffe K. Pharmacological Treatment of Neuropsychiatric Symptoms of Dementia. A Review of the Evidence. JAMA 2005; 293: 596-608

8. Singh S, Wooltorton E. Increased mortality among elderly patients with dementia using atypical antipsychotics. CMA] 2005: 173: 252

9. Wynna ZJ, Cumming JL: Cholinesterase Inhibitor Therapies and Neuropsychiatric Manifestations of Alzheimer's Disease. Dementia and Geriatric Cognitive Disorders 2004; 17: 100-108

\section{Korrespondenzadresse:}

Prof. Dr. med. Jürgen Fritze

Gesundheitspolitischer Sprecher

Deutsche Gesellschaft für Psychiatrie,

Psychotherapie \& Nervenheilkunde (DGPPN)

Asternweg 65

50259 Pulheim 